\title{
Infrared and sub-mm observations of cataclysmic variables
}

\author{
A. Evans \\ Astrophysics Group, Keele University, Keele, Staffordshire, ST5 5BG, UK
}

\section{Introduction}

Although cataclysmic variables (CVs) come in a wide variety of shapes and sizes, the essential ingredients are a compact primary star and a Roche-lobe-filling secondary. In most cases the cool component is a main sequence dwarf, and the compact component a white dwarf (WD). Material from the cool component flows through the inner Lagrangian point via an accretion disc onto the surface of the WD; the flow near the WD is significantly affected by the strength of the magnetic field the WD may have (see Warner for a review of CVs). CVs are characterised by regular eruptions, ranging in energetics and frequency from 'dwarf novae', in which eruptions of amplitude $\sim 3-4 \mathrm{mag}$ in the visual occur every few days to weeks, to classical novae $(\mathrm{CNe})$ in which the eruption is explosive, due to thermonuclear runaway (TNR) in material accreted on the surface of the WD (see Bode \& Evans for a review of $\mathrm{CNe}$ ).

\section{CVs in the infrared}

The cool component and accretion disc dominate the SED of CVs in the near infrared (IR) but in some cases an excess is seen at longer wavelengths. The most likely explanation is emission by dust in the CV system and the origin of this material is problematic. Possibilites include a circumbinary dusty disc, a remnant of the material produced during the common envelope phase of the CV progenitor, and material lost from the CV via winds, the accretion flow or ejected in outbursts (see Brinkworth).

\section{Classical novae}

CNe are a subset of the CVs in which the layer accreted on the WD undergoes TNR. The accreted layer is ejected explosively and some $10^{-4} \mathrm{M}_{\odot}$ of material is ejected at $\sim 1000 \mathrm{~km} \mathrm{~s}^{-1}$; however, there is a long-standing problem in that far more material is ejected in the explosion than seems to be predicted by TNR models, with possible implications for the long-term evolution of CVs. Elemental abundances in the ejecta are highly non-solar, and CNe are expected to be a significant source of ${ }^{13} \mathrm{C},{ }^{17} \mathrm{~F},{ }^{22} \mathrm{Na}$ (which decays to ${ }^{22} \mathrm{Ne}$ ) and ${ }^{26} \mathrm{Al}$ in the Galaxy. A CN eruption goes through an optically thick phase, a free-free phase, a dust-forming phase, a nebular phase and a coronal phase, although not all CNe produce dust, and not all are coronal.

IR spectroscopy of $\mathrm{CNe}$ is key in determining abundances in the ejecta, and for characterising the dust mineralogy in dusty novae. Also, since some of the WD is mixed into the accreted layer and participates in the TNR, the nature of the WD (whether $\mathrm{CO} / \mathrm{ONe}$ ) is also determined by IR spectroscopy; abundances therefore shed light on the TNR and on the nature of the WD. Dustforming CNe display 'chemical dichotomy', in which both O-rich and C-rich dusts are present simultaneously; this arises because CO formation in the ejecta does not go to saturation.

The WD progenitor may have shed some $1 \mathrm{M}_{\odot}$ of material before the binary became a $\mathrm{CV}$, and in some rare cases this material may be seen in the IR and sub-mm in the CN environment.

\section{The recurrent nova RS Oph}

The best studied recurrent nova (RN) is RS Oph, which had its sixth known eruption in 2006. The eruption in RS Oph is also due to TNR on a WD but in this case, the WD is close to the Chandrasekhar limit (and so RS Oph is a candidate Type Ia supernova), and the material ejected in the explosion runs into and shocks the wind of the red giant.

The 2006 eruption was the subject of observations from the X-ray to the radio. Spitzer observations revealed a rich emission line spectrum and yielded a major surprise: the presence of hot 
dust. Unlike $\mathrm{CNe}$, in which dust condenses in the ejecta, the dust in the RS Oph environment must precede the explosion, and is likely located in a circumbinary disc (see Evans et al. for a review of $\mathrm{RNe}$ and the 2006 eruption of $\mathrm{RS} \mathrm{Oph}$ ).

\section{Symbiotic stars}

Symbiotic stars have spectra showing evidence of a hot component (e.g. by virtue of high excitation lines) and an evolved star with prominent $\mathrm{TiO}, \mathrm{VO}$ bands (see Corradi et al.). Many of these systems also undergo nova-like eruptions, and so RS Oph is sometimes classified as a symbiotic. As well as displaying the IR emission and dust features seen in objects like RS Oph and $\mathrm{CNe}$, some also display maser emission. This offers the prospects of measuring orbital motion and even parallax.

\section{Conclusions}

The binarity of CN systems and the kinetics of mass ejection play a major role in shaping the ejecta. Thus, a CN eruption resembles the events that occur during the late phases of evolution of binary stars but with the advantage that the process can be observed in a human lifetime. CNe are important therefore for understanding the shaping of planetary nebulae. The explosions of RS Oph resemble those of supernovae, again in fast-forward.

It is with some justification that $\mathrm{CNe}$ have been described as "unique laboratories in which several poorly-understood astrophysical processes (e.g. mass transfer, common envelope evolution, molecule and grain formation) may be observed in real time."

In the course of the evolution from wide binaries to CVs, a great deal of material is deposited in the interstellar medium. This, together with the mass-loss during eruptions, indicates that they contribute to the chemical evolution of their host galaxies.

\section{Acknowledgements}

I thank the IAU for financial support.

\section{References}

Bode, M. F. \& Evans, A. 2008, eds, Classical Novae, second edition, Cambridge University Press Brinkworth, C. S., et al. 2007, ApJ 659, 1541

Corradi, R. L. M., Mikołajewska, J., Mahoney, T. J. 2003, eds, Symbiotic Stars Probing Stellar Evolution, ASP Conference Series Vol. 303

Evans, A., Bode, M. F., O'Brien, T. J., Darnley, M. J. 2008, eds, RS Ophiuchi (2006) and the Recurrent Nova Phenomenon, ASP Conference Series Vol. 401

Warner, B. 1995, Cataclysmic Variable Stars, Cambridge University Press 